\title{
Influence of volatile anesthesia versus total intravenous anesthesia on chronic postsurgical pain after cardiac surgery using the Initiative on Methods, Measurement, and Pain Assessment in Clinical Trials criteria: study protocol for a prospective randomized controlled trial
}

Hong Yư ${ }^{1}$, Jian-Qiao Zheng ${ }^{1}$, Yu-Si Hua', Shuo-Fang Ren ${ }^{2}$ and Hai Yü ${ }^{1 *}$

\begin{abstract}
Background: Many patients develop chronic postsurgical pain (CPSP) after cardiac surgery, which interferes with their sleep, mood, and quality of life. Studies have suggested that propofol improves postoperative analgesia compared with volatile anesthetics, but its preventive effect on CPSP following cardiac surgery is still unknown. This study compares the incidence of CPSP following cardiac surgery for those receiving volatile anesthesia and those receiving propofol-based total intravenous anesthesia (TIVA) using criteria recommended by the Initiative on Methods, Measurement, and Pain Assessment in Clinical Trials (IMMPACT).

Methods/design: This is a prospective randomized controlled trial. In total, 500 adults undergoing cardiac surgery will be randomly allocated to the volatile or the TIVA group. The volatile group will receive sevoflurane or desflurane during surgery as general anesthesia. The TIVA group will receive propofol-based intravenous agents and no volatile agents during surgery. The primary outcomes will be the frequency of CPSP at 3 months, 6 months, and 1 year after surgery. In this case, CPSP is sternal or thoracic pain. It is defined as either (1) numerical rating scale (NRS) > 0 or (2) meeting all six IMMPACT criteria for CPSP. The IMMPACT criteria are validated pain instruments.

Discussion: To our knowledge, this is the first prospective randomized controlled trial to investigate the prevention of CPSP following cardiac surgery for patients receiving volatile anesthesia compared to those receiving propofolbased TIVA using validated pain instruments in accordance with the IMMPACT recommendations. This study will provide important information on which of these two anesthetic regimens is better for preventing CPSP after cardiac surgery.

Trial registration: Chictr.org.cn, ChiCTR1900020747. Registered on 16 January 2019.

Keywords: Randomized controlled trial, Volatile anesthesia, Propofol, Total intravenous anesthesia (TIVA), Chronic postsurgical pain (CPSP), Initiative on methods, Measurement and Pain Assessment in Clinical Trials (IMMPACT) criteria assessment
\end{abstract}

\footnotetext{
* Correspondence: yuhaishan117@yahoo.com

'Department of Anesthesiology, West China Hospital, Sichuan University \& The Research Units of West China (2018RU012), Chinese Academy of Medical Sciences, Chengdu 610041, People's Republic of China

Full list of author information is available at the end of the article
}

(c) The Author(s). 2019 Open Access This article is distributed under the terms of the Creative Commons Attribution 4.0 International License (http://creativecommons.org/licenses/by/4.0/), which permits unrestricted use, distribution, and reproduction in any medium, provided you give appropriate credit to the original author(s) and the source, provide a link to the Creative Commons license, and indicate if changes were made. The Creative Commons Public Domain Dedication waiver (http://creativecommons.org/publicdomain/zero/1.0/) applies to the data made available in this article, unless otherwise stated. 


\section{Background}

Cardiac surgery is one of the most common forms of major surgery, with over 2 million patients undergoing this procedure worldwide each year [1]. Many patients develop chronic postsurgical pain (CPSP), which can occur in the anterior thorax after a median sternotomy [1]. It is estimated that CPSP has an incidence of $11-56 \%$ in patients undergoing cardiac or thoracic surgery, depending on the study population and length of follow-up [2-5]. CPSP that persists after cardiac surgery is a major clinical problem, because it disturbs daily life and interferes with sleep, mood, and quality of life [6-8]. Considering the large number of patients who undergo cardiac surgery, identifying potential treatments for CPSP is important [9]. In addition to standard postoperative analgesics, it has been suggested that corticosteroids, N-methyl-D-aspartate (NMDA) antagonists, alpha-2 agonists, local anesthetics, and gabapentinoids can reduce the risk of CPSP after cardiac surgery [5]. However, no specific therapy has been demonstrated to protect against CPSP [10-12].

Patients undergoing cardiac surgery need general anesthesia from either intravenous (IV) anesthetics (such as propofol) or volatile anesthetics (such as isoflurane, sevoflurane, and desflurane). Studies have found that propofol has anti-inflammatory and antioxidative effects on the biosynthesis of cytokines, which are important in pain signaling [13]. Propofol's ability to scavenge free radicals is useful and important. It has antioxidant properties and may also dynamically protect the body [14] Moreover, propofol can modulate NMDA receptors in neurons in vivo, which play a crucial role in the transmission and maintenance of the pain signaling pathway $[14,15]$. These anti-inflammatory, free radical scavenging, and NMDA receptor antagonistic properties of propofol imply that it may have a possible perioperative analgesic effect. The meta-analyses by Qiu et al. and Peng et al. suggest that propofol improved postoperative analgesia compared with inhalational anesthesia [16, 17]. However, most previous studies were not designed to detect differences in chronic pain $[16,17]$, and a few clinical trials investigating CPSP focused on non-cardiac surgery $[18,19]$. To date, no published clinical trial has compared the effects of volatile anesthesia with those of propofolbased total intravenous anesthesia (TIVA) in preventing CPSP after cardiac surgery through sternotomy.

Prior studies focusing on CPSP after either cardiac or non-cardiac surgery assessed pain using a yes or no scoring system or a numerical rating scale (NRS) to evaluate the level of pain. Few studies have used the Initiative on Methods, Measurement, and Pain Assessment in Clinical Trials (IMMPACT) recommendations to evaluate CPSP [20]. The IMMPACT approach to assessing CPSP evaluates the quality of the pain, the degree of pain at rest and during movement, the clinical meaningfulness of the pain, and the influence of pain on physical and emotional functioning, instead of focusing on only the absence or presence of pain [20-23]. Thus, the aim of this randomized controlled trial is to assess the prevention of CPSP following cardiac surgery through sternotomy for patients receiving volatile anesthesia compared to those receiving propofol-based TIVA using validated pain instruments in accordance with the IMMPACT recommendations.

\section{Methods/design}

\section{Study design, approval, and registration}

The planned study is a parallel-group randomized controlled trial with a 1:1 allocation ratio undertaken in West China Hospital of Sichuan University. Figure 1 is the trial flowchart. Recruitment commenced in February 2019. Additional file 1 is the Standard Protocol Items: Recommendations for Interventional Trials (SPIRIT) checklist. The schedule of enrollment, interventions, and assessments follows the SPIRIT Figure (Additional file 2). The study has been approved by the ethics committee of West China Hospital of Sichuan University and has been prospectively registered at Chictr.org.cn (ChiCTR1900020747).

\section{Study aim}

The aim of our study is to compare the incidence of CPSP following cardiac surgery for patients receiving volatile anesthesia and those receiving propofol-based TIVA using the IMMPACT criteria.

\section{Participants}

We plan to enroll 500 participants aged more than 18 years undergoing a cardiopulmonary bypass (CPB) for any elective cardiac surgical procedure via a median sternotomy such as procedures that involve the valves, coronary arteries, or aorta, or combined procedures.

\section{Inclusion criteria}

Participants must meet all the inclusion criteria:

1. Aged older than 18 years

2. Undergoing a $\mathrm{CPB}$ for cardiac surgery via a median sternotomy

3. Signed the informed consent form

\section{Exclusion criteria}

Patients who meet any of the following criteria will be excluded from participation:

1. Those undergoing combined cardiac and noncardiac surgery

2. Those undergoing emergency surgery

3. Pregnant women

4. Those with a suspected family history of malignant hyperthermia or propofol infusion syndrome 


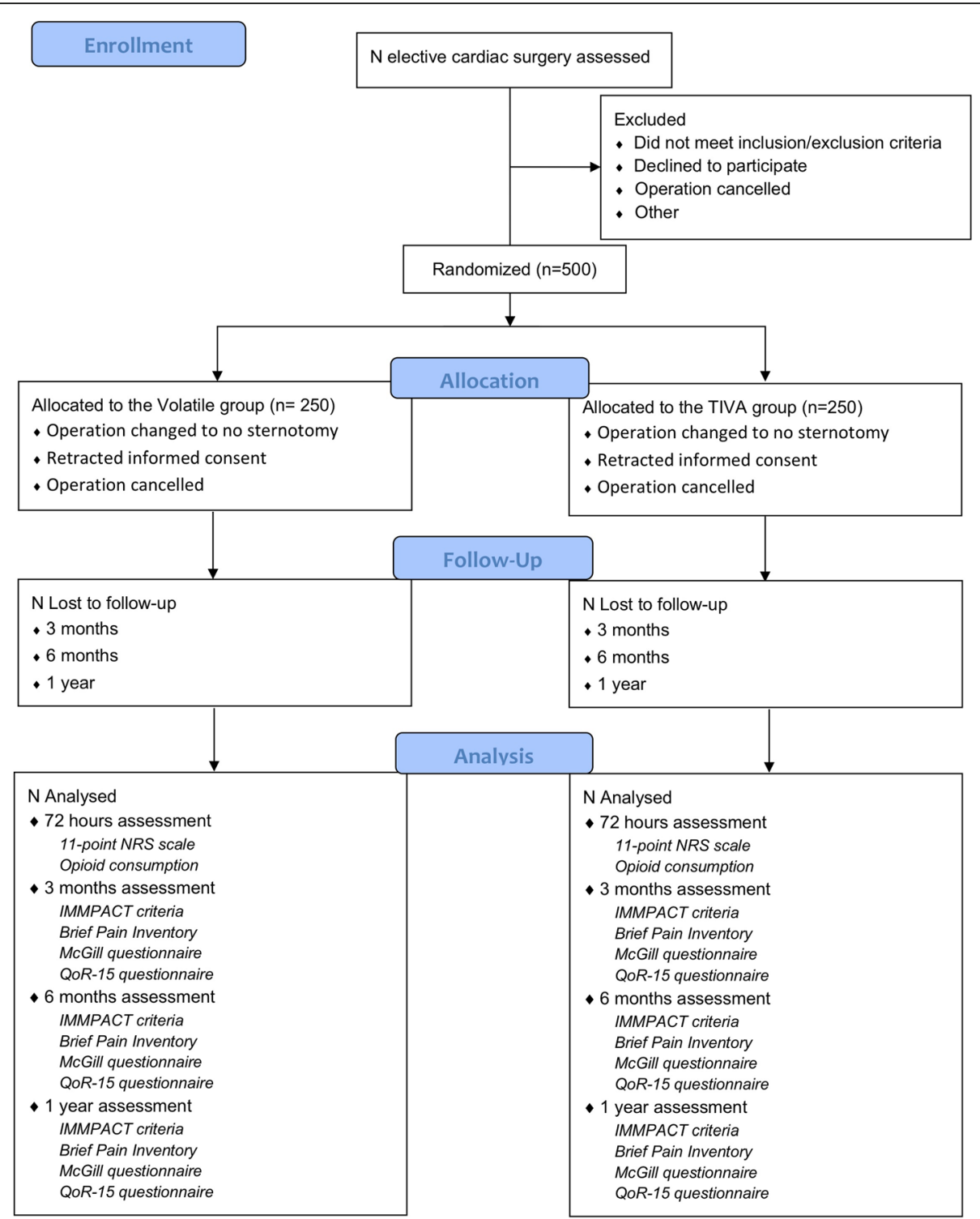

Fig. 1 Consort diagram of study participant flow. TIVA total intravenous anesthesia, NRS numeric rating scale, IMMPACT Initiative on Methods, Measurement, and Pain Assessment in Clinical Trials, QoR-15 15-item Quality of Recovery Questionnaire

5. Those who fail to cooperate with the follow-up procedures due to a lack of understanding of the study procedures

\section{Randomization, allocation, and concealment}

Once informed consent has been received and the preoperative assessments completed, patients will be entered into the trial. Subjects will be allocated using a web-based centralized dynamic randomization service. The dynamic randomization considers patient characteristics including age, gender, European System for Cardiac Operative Risk Evaluation (EuroSCORE) score, predicted CPB duration, and body mass index. The anesthesiologists will be aware of patients' group allocation because they will provide the trial intervention, but they will not be involved in either the postoperative treatment or the analysis. Patients, intensive care physicians, data collectors, and outcome adjudicators are blinded to treatment allocation.

\section{Interventions}

Patients who meet the enrollment criteria will be randomized 1:1 to either the volatile or the TIVA group. Three investigators (Hong Yu, JQZ, and YSH) will explain the treatment intervention in detail and supervise the compliance of the intervention throughout the entire procedure (from maintenance of anesthesia to transport to an intensive care unit). 


\section{The volatile group}

The volatile group will receive sevoflurane or desflurane to provide general anesthesia during surgery from maintenance of anesthesia to transport to an intensive care unit, including during $\mathrm{CPB}$. Maintenance of anesthesia in the treatment group uses sevoflurane or desflurane at a minimum end-tidal concentration of 0.5-2 minimal alveolar concentration throughout the entire procedure. During CPB, patients will receive sevoflurane or desflurane from a vaporizer connected to an air blender, which is connected to an oxygenator. The minimal alveolar concentration is measured at the outlet of the oxygenator of the extracorporeal circulation.

\section{The TIVA group}

The TIVA group will receive propofol at an infusion rate of $3-8 \mathrm{mg} \mathrm{kg}^{-1} \mathrm{~h}^{-1}$ with or without other IV agents. The only absolute criterion for this group is that no volatile anesthetic is to be used at any time during the procedure.

\section{Perioperative management Induction of anesthesia}

General anesthesia will be induced with midazolam, sufentanil, and propofol as necessary. Tracheal intubation will be facilitated with either rocuronium or cisatracurium. There is no restriction on the type or dose of anesthetic used to induce anesthesia.

\section{Ventilation}

Patients will be ventilated using a lung-protecting ventilation strategy before and after $\mathrm{CPB}$. The pressure-controlled ventilation will maintain a tidal volume of $6-8 \mathrm{ml} \mathrm{kg}^{-1}$ based on ideal body weight, a positive end expiratory pressure of 5-8 $\mathrm{cm} \mathrm{H}_{2} \mathrm{O}$, an inspiratory to expiratory ratio of $1: 2$; an inspired oxygen fraction of 0.4 to 0.8 , and a respiratory rate of $10-16$ per min adjusted to keep a desired endtidal $\mathrm{CO}_{2}$ of $35-45 \mathrm{mmHg}$. A recruitment maneuver with a peak airway pressure of $30 \mathrm{cmH}_{2} \mathrm{O}$ for $30 \mathrm{~s}$, as an essential part of the protective ventilation strategy, will be performed before beginning and before discontinuing $\mathrm{CPB}$ and exiting from the operating room. The use of ventilation during $\mathrm{CPB}$ will be chosen by the anesthesia care providers.

\section{Anesthesia maintenance}

Propofol or inhalation anesthetics plus sufentanil and a nondepolarizing muscle relaxant will be used to maintain general anesthesia with dosages at the discretion of the attending clinicians. Sufentanil will be administered to avoid changes of mean arterial pressure of more than $20 \%$ from baseline while maintaining a mean arterial pressure of at least $65 \mathrm{mmHg}$. A vasopressor will be administered as necessary. Remifentanil will be administered at an infusion rate of $0.1-0.2 \mu \mathrm{g} \mathrm{kg}^{-1} \mathrm{~min}^{-1}$. The dosage of dexmedetomidine will be limited to less than $0.5 \mu \mathrm{g} \mathrm{kg}^{-1} \mathrm{~h}^{-1}$ if needed. No antiemetics will be administrated for nausea or vomiting prophylaxis.

\section{Postoperative analgesia}

After surgery, patients will be transferred to an intensive care unit for further care. Patients will receive an infusion of $10-25 \mu \mathrm{g} \mathrm{kg}^{-1} \mathrm{~h}^{-1}$ morphine or $100 \mathrm{mg}$ of IV meperidine to maintain an NRS of less than $4(0=$ no pain, $10=$ worst pain imaginable), as assessed by the nursing personnel. IV analgesia will be discontinued, and patients will be given oral celecoxib or ibuprofen when they can tolerate oral medication. Patient-controlled IV analgesia pumps will not be used.

\section{Data collection}

\section{Baseline characteristics of patients}

Demographic data, cardiac history, coexisting medical conditions, comorbidities, smoking status, EuroSCORE score, depression and anxiety history, chronic pain at presentation in an area other than the operative site, surgical procedure, intraoperative sufentanil and remifentanil dosage, and health-related quality of life measured with the 15-item Quality of Recovery Questionnaire (QoR-15) [24] will be recorded.

\section{Acute pain assessment at 24,48 , and $72 \mathrm{~h}$ after surgery}

Patients will be visited and evaluated over the first $72 \mathrm{~h}$ after surgery. Pain is assessed using an 11-point NRS scale $(0=$ no pain, $10=$ worst pain imaginable $)$ at 24,48 , and 72 $\mathrm{h}$ after surgery. The amount of opioid analgesics consumed is determined from their electronic medical record and is converted to an equivalent dose of IV morphine [25].

\section{Follow-ups at 3 months, 6 months, and 1 year}

Each patient will receive follow-up phone calls at 3 months, 6 months, and 1 year after surgery to answer questions regarding the presence, quality, and severity of pain using the Brief Pain Inventory [26], the McGill Short Form Questionnaire [27], and the QoR-15 questionnaire [24]. Each patient will leave at least three phone numbers and receive a maximum of three telephone calls if contact could not be made.

\section{Outcomes}

\section{Primary outcome}

The primary outcome is the occurrence of CPSP at 3 months, 6 months, and 1 year after surgery. CPSP is defined as sternal or thoracic pain that the patient identifies as related to their surgery in two ways: (1) NRS $>0$ and (2) pain that meets all six IMMPACT criteria for CPSP. The IMMPACT criteria are validated instruments for assessing pain [28]. 


\section{IMMPACT Questionnaires}

Chronic pain is assessed in accordance with the IMMPACT recommendations in the following six domains [28]: (1) absence or presence of pain in the area of surgery, (2) clinically important daily average pain (NRS $\geq 4$ on a 0 - to 10 -point scale), (3) clinically important pain at rest (NRS $\geq 4$ ), (4) clinically important pain intensity upon movement or activity (NRS $\geq 4$ ), (5) quality of pain, and (6) physical and emotional functioning $[21,22,29]$. The Brief Pain Inventory is used to determine the domains 1 to 4 and 6 . It measures the intensity of daily average pain, pain at rest, pain during movement or activity, as well as physical and emotional functioning [26]. The McGill Pain Questionnaire is used to assess domain 5, which relates to the impact of both sensory and affective pain [27]. A total pain index score $\geq 12$ is associated with chronic pain [30]. Subjects have to meet the threshold for all six outcome domains to fulfill the IMMPACT criteria.

\section{Secondary outcomes}

The secondary outcomes are: (1) NRS pain scores (010) 24,48 , and $72 \mathrm{~h}$ after surgery, (2) opioid consumption during the first $72 \mathrm{~h}$ after surgery, (3) the Brief Pain Inventory, the McGill Pain Questionnaire, and healthrelated quality of life measured with QoR-15 at 3 months, 6 months, and 1 year after surgery.

\section{Statistics}

\section{Sample size estimate}

The sample selected for this study was based on the finding of our prior study exploring the incidence and possible risk factors of CPSP in patients undergoing cardiac surgery with $\mathrm{CPB}$ via a median sternotomy. That study showed that $60.9 \%$ patients had CPSP at 3 months postoperatively [31]. The sample size for the current study is 250 patients per group, for a total of 500 patients. The study has $80-90 \%$ power to detect a 25 relative risk reduction for the primary outcome of CPSP at 3 months at a significance level (alpha) of 0.05 (two-sided), anticipating a 50$60 \%$ CPSP rate in the control (inhalation or TIVA) arm with an allowance of $10 \%$ of patients lost to follow-up, or withdrawn or withdrawing from the study.

\section{Statistical analyses}

Data will be expressed as means \pm standard deviation or numbers (percentages). Baseline characteristics will be compared using chi-squared or Fisher's exact tests, a Student's $t$ test, or a nonparametric test as appropriate. The primary outcome, the occurrence of CPSP at 3 months, 6 months, and 1 year after surgery, will be compared using chi-squared or Fisher's exact tests, and the relative risks and the $95 \%$ confidence interval will be calculated. All analyses of primary outcomes will be conducted using the intention-to-treat approach. A sensitivity analysis using the per-protocol approach will also be performed. In addition, a multiple logistic regression analysis will be used to identify relevant baseline covariates associated with the primary outcome. Variables tested in the model will be selected if $P<0.10$ or if they are clinically relevant (such as usage of analgesics during surgery). All secondary outcomes are continuous variables and will be compared using the unequal-variance Student's $t$ test. Results are considered statistically significant if $P<0.05$. Statistical analyses are performed using statistical software SPSS 17.0.

\section{Participant timeline}

Recruitment of patients and data collection started in February 2019. Sufficient participants have been enrolled (500 patients) at the end of June 2019. The 1-year postoperative follow-up will be completed in June 2020.

\section{Data management and monitoring}

All original data will be recorded in case report forms. The study supervisor (Hai $\mathrm{Yu}$ ) will supervise the conduct of the trial conduction and perform monthly audits of the trial.

\section{Discussion}

To our knowledge, this is the first prospective randomized controlled trial to investigate the prevention of CPSP following cardiac surgery through sternotomy for patients receiving volatile anesthesia compared to those receiving propofol-based TIVA using validated pain instruments in accordance with the IMMPACT recommendations.

CPSP is defined as persistent or recurrent pain lasting longer than 3 months and without an apparent cause [32]. Our results are clinically important because the incidence of CPSP can occur in $11-56 \%$ of patients undergoing cardiac or thoracic surgery with a median sternotomy, and currently there are established methods of prevention [10-12]. As we know, general anesthesia is routinely used in cardiac surgery. To date, it is well established that propofol can improve postoperative analgesia compared with inhalational anesthesia $[16,17]$. As acute pain is viewed as an initial phase of the pain response that has the potential to progress to chronic pain [5], it was assumed that propofol has the potential to prevent CPSP, though it has conflicting roles. Ogurlu et al. reported that general anesthesia with propofol was associated with reduced persistent pain at 3 months compared to sevoflurane-based anesthesia, among patients undergoing open abdominal hysterectomy [18]. Moreover, Song et al. found consistent results in patients who had undergone a thoracotomy during surgery for lung and esophageal cancer [19].

However, there is no information in the literature on the relationship between the type of anesthesia used in cardiac surgery and subsequent chronic pain. To date, 
no clinical trial has been published comparing the effects of volatile anesthesia with those of propofol-based TIVA on postoperative acute and chronic pain after cardiac surgery through sternotomy.

On the other hand, prior studies focusing on CPSP after either cardiac or non-cardiac surgery assessed pain using a yes or no scoring system or the NRS evaluation of the intensity of pain. Few studies have used the IMMPACT recommendations [20]. IMMPACT is a consortium of researchers and practitioners in pain medicine whose mission is to develop consensus reviews and recommendations for improving the design, execution, and interpretation of clinical trials of treatments for pain [33]. The domains of pain recommended by IMMPACT show the diversified effect of anesthetics on pain at rest, pain during activity, the quality of pain, and the physical and emotional impact of pain.

In summary, this randomized controlled trial may provide important information on the influence of these two anesthetic regimens on CPSP after cardiac surgery. Moreover, using validated pain instruments to measure CPSP in accordance with the IMMPACT recommendations will add to the currently available data on CPSP, which is important since CPSP is clinically meaningful and disturbs patients' physical and emotional functioning and quality of life.

\section{Supplementary information}

Supplementary information accompanies this paper at https://doi.org/10. 1186/s13063-019-3742-4.

Additional file 1. SPIRIT 2013 checklist.

Additional file 2. SPIRIT figure.

\section{Abbreviations \\ CPB: Cardiopulmonary bypass; CPSP: Chronic postsurgical pain; EuroSCORE: European System for Cardiac Operative Risk Evaluation; IMMPACT: Initiative on Methods, Measurement, and Pain Assessment in Clinical Trials; IV: Intravenous; NMDA: N- methyl-D-aspartate; NRS: Numerical rating scale; QoR-15: 15-item Quality of Recovery Questionnaire: SPIRIT: Standard Protocol Items: Recommendations for Interventional Trials; TIVA: Total intravenous anesthesia}

\section{Acknowledgements}

We thank the cardiovascular anesthesiology team and the perfusion team in West China Hospital of Sichuan University for their continued support, and the patients and staff at the Department of Cardiovascular Surgery for their assistance throughout this study.

\section{Trial status}

The current protocol is version 1.0 and was issued on 1 January 2018 Recruitment of patients and data collection started in February 2019. Sufficient participants have been enrolled (500 patients) at the end of June 2019. The 1-year postoperative follow-up will be completed in June 2020.

\section{Authors' contributions}

Hai Yu (HY1) conceptualized, resourced, and supervised the study, and reviewed and edited the manuscript. YSH was responsible for data curation and undertook the formal analysis. HY1 and Hong Yu (HY2) were responsible for acquiring funding, developing the methodology, and administering the project. HY2, YSH, JQZ, and SFR undertook the investigation. SFR wrote the software. HY2, JQZ, and YSH validated the data. JQZ and YSH visualized the data. HY2 wrote the original draft of this manuscript. All authors read and approved the final version of the manuscript.

\section{Funding}

This research is supported by a Young Scholar Research Grant from the Chinese Anesthesiologist Association (312180142). The Chinese Anesthesiologist Association will not intervene in any aspect of the trial, including its design, data collection, analysis, or presentation.

Availability of data and materials

Not applicable.

Ethics approval and consent to participate

The study protocol has been approved by the ethics committee of West China Hospital of Sichuan University (the first version of this protocol was approved on 16 January 2019, reference 2018[562]). We will obtain written informed consent from each patient before they are randomized.

Participants may withdraw from the trial at any time for any reason.

\section{Consent for publication}

Not applicable.

\section{Competing interests}

The authors declare that they have no competing interests.

\section{Author details}

${ }^{1}$ Department of Anesthesiology, West China Hospital, Sichuan University \& The Research Units of West China (2018RU012), Chinese Academy of Medical Sciences, Chengdu 610041, People's Republic of China. ${ }^{2}$ Department of Cardiovascular surgery, West China Hospital, Sichuan University \& The Research Units of West China (2018RU012), Chinese Academy of Medical Sciences, Chengdu 610041, People's Republic of China.

Received: 31 March 2019 Accepted: 23 September 2019

Published online: 27 November 2019

\section{References}

1. Costa MA, Trentini CA, Schafranski MD, Pipino O, Gomes RZ, Reis ES. Factors Associated With the Development of Chronic Post-Sternotomy Pain: a CaseControl Study. Braz J Cardiovasc Surg. 2015;30(5):552-6.

2. van Gulik L, Janssen LI, Ahlers SJ, Bruins P, Driessen AH, van Boven WJ, van Dongen EP, Knibbe CA. Risk factors for chronic thoracic pain after cardiac surgery via sternotomy. Eur J Cardiothoracic Surg. 2011;40(6):1309-13.

3. Page MG, Watt-Watson J, Choiniere M. Do depression and anxiety profiles over time predict persistent post-surgical pain? A study in cardiac surgery patients. Eur J Pain (London, England). 2017;21 (6):965-76.

4. Mazzeffi M, Khelemsky Y. Poststernotomy pain: a clinical review. J Cardiothorac Vasc Anesth. 2011;25(6):1163-78.

5. Gjeilo KH, Stenseth R, Klepstad P. Risk factors and early pharmacological interventions to prevent chronic postsurgical pain following cardiac surgery. Am J Cardiovasc Drugs. 2014;14(5):335-42.

6. Taillefer MC, Carrier M, Belisle S, Levesque S, Lanctot H, Boisvert AM, Choiniere M. Prevalence, characteristics, and predictors of chronic nonanginal postoperative pain after a cardiac operation: a cross-sectional study. J Thorac Cardiovasc Surg. 2006;131(6):1274-80.

7. Li M, Zhang M, Wang H, Pan X, Wu W, Zhang Q, Liu Y, Zhang H. The Efficacy of Internet-based Intervention on Quality of Life for Patients with Chronic Post-surgical Pain. Iran J Public Health. 2016;45(12):1604-9.

8. Jensen MP, Chodroff MJ, Dworkin RH. The impact of neuropathic pain on health-related quality of life: review and implications. Neurology. 2007; 68(15):1178-82.

9. Scholz J, Yaksh TL. Preclinical research on persistent postsurgical pain: what we don't know, but should start studying. Anesthesiology. 2010; 112(3):511-53.

10. de Hoogd S, Ahlers S, van Dongen EPA, van de Garde EMW, Daeter EJ, Dahan A, Tibboel D, Knibbe CAJ. Randomized controlled trial on the influence of intra-operative remifentanil versus fentanyl on acute and chronic pain after cardiac surgery. Pain Pract. 2018;18(4):443-51. 
11. Peyton PJ, Wu C, Jacobson T, Hogg M, Zia F, Leslie K. The effect of a perioperative ketamine infusion on the incidence of chronic postsurgical pain-a pilot study. Anaesth Intensive Care. 2017:45(4):459-65.

12. Turan A, Belley-Cote EP, Vincent J, Sessler DI, Devereaux PJ, Yusuf S, van Oostveen R, Cordova G, Yared JP, Yu H, et al. Methylprednisolone Does Not Reduce Persistent Pain after Cardiac Surgery. Anesthesiology. 2015;123(6):1404-10.

13. Chen RM, Chen TG, Chen TL, Lin LL, Chang CC, Chang HC, Wu CH. Antiinflammatory and antioxidative effects of propofol on lipopolysaccharideactivated macrophages. Ann N Y Acad Sci. 2005;1042:262-71.

14. Li W, Zhang Y, Liu Y, Yue F, Lu Y, Qiu H, Gao D, Gao Y, Wu Y, Wang Z, et al. In vitro kinetic evaluation of the free radical scavenging ability of propofol. Anesthesiology. 2012;116(6):1258-66.

15. Latremoliere A, Woolf CJ. Central sensitization: a generator of pain hypersensitivity by central neural plasticity. J Pain. 2009;10(9):895-926.

16. Qiu Q, Choi SW, Wong SS, Irwin MG, Cheung CW. Effects of intraoperative maintenance of general anaesthesia with propofol on postoperative pain outcomes - a systematic review and meta-analysis. Anaesthesia. 2016;71(10):1222-33.

17. Peng K, Liu HY, Wu SR, Liu H, Zhang ZC, Ji FH. Does Propofol Anesthesia Lead to Less Postoperative Pain Compared With Inhalational Anesthesia?: A Systematic Review and Meta-analysis. Anesth Analg. 2016;123(4):846-58.

18. Ogurlu M, Sari S, Kucuk M, Bakis M, Ugur B, Eshraghi YE, Galimberti F, Turan A. Comparison of the effect of propofol and sevoflurane anaesthesia on acute and chronic postoperative pain after hysterectomy. Anaesth Intensive Care. 2014;42(3):365-70.

19. Song JG, Shin JW, Lee EH, Choi DK, Bang JY, Chin JH, Choi IC. Incidence of post-thoracotomy pain: a comparison between total intravenous anaesthesia and inhalation anaesthesia. Eur J Cardiothoracic Surg. 2012; 41(5):1078-82.

20. Gewandter JS, Dworkin RH, Turk DC, Farrar JT, Fillingim RB, Gilron I, Markman JD, Oaklander AL, Polydefkis MJ, Raja SN, et al. Research design considerations for chronic pain prevention clinical trials: IMMPACT recommendations. Pain. 2015;156(7):1184-97.

21. Dworkin RH, Turk DC, Peirce-Sandner S, Baron R, Bellamy N, Burke LB, Chappell A, Chartier K, Cleeland CS, Costello A, et al. Research design considerations for confirmatory chronic pain clinical trials: IMMPACT recommendations. Pain. 2010;149(2):177-93.

22. Dworkin RH, Turk DC, Peirce-Sandner S, Burke LB, Farrar JT, Gilron I, Jensen MP, Katz NP, Raja SN, Rappaport BA, et al. Considerations for improving assay sensitivity in chronic pain clinical trials: IMMPACT recommendations. Pain. 2012;153(6):1148-58.

23. Taylor AM, Phillips K, Patel KV, Turk DC, Dworkin RH, Beaton D, Clauw DJ, Gignac MA, Markman JD, Williams DA, et al. Assessment of physical function and participation in chronic pain clinical trials: IMMPACT/OMERACT recommendations. Pain. 2016;157(9):1836-50.

24. Stark PA, Myles PS, Burke JA. Development and psychometric evaluation of a postoperative quality of recovery score: the QoR-15. Anesthesiology. 2013; 118(6):1332-40

25. Macintyre PE, Ready LB. Pharmacology of opioids, Acute Pain ManagementA Practical Guide. 2nd ed; 2001.

26. Daut RL, Cleeland CS, Flanery RC. Development of the Wisconsin Brief Pain Questionnaire to assess pain in cancer and other diseases. Pain. 1983;17(2): 197-210.

27. Dudgeon D, Raubertas RF, Rosenthal SN. The short-form McGill Pain Questionnaire in chronic cancer pain. J Pain Symptom Manag. 1993; 8(4):191-5.

28. Gewandter JS, Dworkin RH, Turk DC, McDermott MP, Baron R, Gastonguay MR, Gilron I, Katz NP, Mehta C, Raja SN, et al. Research designs for proof-ofconcept chronic pain clinical trials: IMMPACT recommendations. Pain. 2014; 155(9):1683-95.

29. Dworkin RH, Turk DC, Farrar JT, Haythornthwaite JA, Jensen MP, Katz $N P$, Kerns RD, Stucki G, Allen RR, Bellamy N, et al. Core outcome measures for chronic pain clinical trials: IMMPACT recommendations. Pain. 2005;113(1-2):9-19.

30. Wilkie DJ, Savedra MC, Holzemer WL, Tesler MD, Paul SM. Use of the McGill Pain Questionnaire to measure pain: a meta-analysis. Nurs Res. 1990;39(1):36-41.

31. Zhang YHZM, Yu H. Incidence and possible risk factors of chronic postsurgical pain after cardiac surgery: A prospective cohort study. Chin J Clin Thorac Cardiovasc Surg. 2018;25(8):711-4.
32. Treede RD, Rief W, Barke A, Aziz Q, Bennett MI, Benoliel R, Cohen M, Evers S, Finnerup NB, First MB, et al. A classification of chronic pain for ICD-11. Pain. 2015;156(6):1003-7.

33. Kendall MC, McCarthy RJ, Panaro S, Goodwin E, Bialek JM, Nader A, De Oliveira GS, Jr. The effect of intraoperative systemic lidocaine on postoperative persistent pain using Initiative on Methods, Measurement, and Pain Assessment in Clinical Trials criteria assessment following breast cancer surgery: a randomized, double-blind, placebo-controlled trial. Pain Pract. 2018:18(3):350-9.

\section{Publisher's Note}

Springer Nature remains neutral with regard to jurisdictional claims in published maps and institutional affiliations.
Ready to submit your research? Choose BMC and benefit from:

- fast, convenient online submission

- thorough peer review by experienced researchers in your field

- rapid publication on acceptance

- support for research data, including large and complex data types

- gold Open Access which fosters wider collaboration and increased citations

- maximum visibility for your research: over $100 \mathrm{M}$ website views per year

At BMC, research is always in progress.

Learn more biomedcentral.com/submissions 